Revista Brasileira de Agricultura Irrigada v.8, nº.6, p.444 - 454, 2014

ISSN 1982-7679 (On-line)

Fortaleza, CE, INOVAGRI - http://www.inovagri.org.br

DOI: $10.7127 /$ rbai.v8n600254

Protocolo 254.14 - 31/05/2014 Aprovado em 30/09/2014

\title{
COEFICIENTES DE UNIFORMIDADE EM SISTEMA DE IRRIGAÇÃO POR GOTEJAMENTO
}

\author{
Fernando Nobre Cunha ${ }^{1}$, Nelmício Furtado da Silva ${ }^{2}$, Marconi Batista Teixeira ${ }^{3}$, José \\ Joaquim de Carvalho ${ }^{4}$, Luciana Minervina de Freitas Moura ${ }^{5}$, Cláudio Carvalho dos \\ Santos ${ }^{6}$
}

\section{RESUMO}

A uniformidade de distribuição é fundamental na avaliação do desempenho de sistemas de irrigação, sendo que a utilização do coeficiente correto torna-se fator decisivo. Objetivouse neste estudo demonstrar a discriminação dos coeficientes de uniformidade em sistema de gotejamento em condição superficial e subsuperficial. O experimento foi conduzido em bancada de ensaio instalada em casa de vegetação na área experimental do Instituto Federal de Educação, Ciência e Tecnologia Goiano - Campus Rio Verde. O delineamento experimental utilizado foi inteiramente casualizado analisado em esquema de parcelas subdividas 6 × 20, com 10 repetições, as parcelas foram compostas por seis distribuições de partículas sólidas e as subparcelas 20 ensaios. A unidade experimental foi constituída de recipientes de PVC com as dimensões de 0,1 m x 0,6 m (D x C), contendo cada recipiente um gotejador. Foram efetuados os cálculos da vazão e de uniformidade de aplicação de água utilizando-se os coeficientes de Christiansen (CUC), estatístico (CUE), distribuição (CUD), absoluto (CUA), Hart (CUH) e eficiência padrão da HSPA (UDH). O CUA foi mais discriminante em condição superficial enquanto o CUC teve maior discriminação em condição subsuperficial. Os coeficientes quando comparado entre si, em ordem decrescente, apresentaram a seguinte classificação dos percentuais de discriminação superficialmente: CUA, CUC, CUE, CUH, UDH e CUD; já subsuperficialmente CUC, CUA, CUH, CUE, UDH e CUD.

Palavras-chave: irrigação localizada, uniformidade de distribuição, manejo da irrigação.

\section{COEFFICIENT OF UNIFORMITY IN DRIP IRRIGATION SYSTEM}

\footnotetext{
${ }^{1}$ Doutorando em Ciências Agrárias - Agronomia, Instituto Federal Goiano - Campus Rio Verde, Rodovia Sul Goiana, Km 01, CEP: 75.901-170, Rio Verde - GO, e-mail: fernandonobrecunha@hotmail.com

${ }^{2}$ Doutorando em Ciências Agrárias - Agronomia, IFGoiano - Campus Rio Verde, e-mail: nelmiciofurtado@gmail.com

${ }^{3}$ Eng. Agrônomo, Prof. Dr. em Agronomia, IFGoiano - Campus Rio Verde, e-mail: marconibt@gmail.com

${ }^{4}$ Pós-doutorando em Ciências Agrárias - Agronomia, IFGoiano - Campus Rio Verde, e-mail: josejoaquimcarvalho@yahoo.com.br

${ }^{5}$ Mestranda em Ciências Agrárias - Agronomia, IFGoiano - Campus Rio Verde, e-mail: lucianaminervina@gmail.com

${ }^{6}$ Graduando em Agronomia, IFGoiano - Campus Rio Verde, e-mail: santos.claudiocarvalho@gmail.com
} 


\begin{abstract}
The distribution uniformity is critical in assessing the performance of irrigation systems, and the use of correct method becomes deciding factor. The objective of this study demonstrate the discrimination of uniformity of water application for different methods in surface drip system and subsurface. The experiment was conducted in a bench trial in a greenhouse installed in the experimental area of the Federal Institute of Education, Science and Technology Goiano - Campus Rio Verde. The experimental design was completely randomized analysis in scheme of split plot 6 x 20 with 10 repetitions, composed six plots distributions of solid particles and subplots 20 trials. The experimental unit consisted of PVC containers with dimensions of $0.1 \mathrm{~m} \times 0.6 \mathrm{~m}$ ( $\mathrm{D} \times \mathrm{C}$ ), each container containing a dripper. Were made the Calculations of flow and uniformity of water application using the coefficients Christiansen (CUC), statistical (CUE), distribution (CUD), absolute (CUA), Hart (CUH) and standard efficiency of HSPA (UDH). The CUA was most discriminating in surface condition while CUC had greater discrimination in subsurface condition. The coefficients compared to each other, in descending order, presented the following classification of the percentage of discrimination superficially: CUA, CUC, CUE, CUH, UDH and CUD, already subsurface CUC, CUA, CUH, CUE, UDH and CUD.
\end{abstract}

Keywords: localized irrigation, distribution uniformity, irrigation management.

\section{INTRODUÇÃO}

Os sistemas de irrigação localizada são de grande importância no cenário agrícola brasileiro, com aplicações voltadas principalmente para a fruticultura, horticultura e fertirrigação (CARVALHO \& OLIVEIRA, 2012).

Este sistema pode ser utilizado de duas formas: na superfície ou enterrado, também chamado sistema de irrigação subsuperficial (BARROS et al. 2009).

Este método tem sido bastante utilizado devido suas características de aplicações de pequenas vazões e alta frequência, aplicação de fertilizantes via água de irrigação (distribuídos na zona radicular das culturas), baixas pressões e alta eficiência (geralmente superior a 90 \%), possibilitando um controle eficiente da lâmina de irrigação (BERNARDO et al., 2008).

Vale lembrar que a distribuição da água aplicada dificilmente será plenamente uniforme, e a mensuração dessa variabilidade é fundamental na avaliação do desempenho da irrigação (SILVA et al., 2004). Segundo Barreto Filho et al. (2000), a uniformidade de aplicação de água é um parâmetro que caracteriza o sistema de irrigação em função da diferença de volume aplicado na planta ao longo das linhas laterais e de acordo com Faria et al. (2004), a uniformidade de aplicação de água pelos gotejadores é fator fundamental ao sucesso do empreendimento.

Para Mantovani et al. (2009) a avaliação da irrigação é uma importante etapa para obter as informações relacionadas à eficiência de uso da água do sistema de irrigação, perdas durante a aplicação e uniformidade de distribuição de água, funcionamento real do sistema (vazão, pressão, lâmina e entupimento) e necessidade de manutenção.

O coeficiente de uniformidade de distribuição da água e a eficiência de aplicação são os principais parâmetros utilizados, pois expressam a qualidade da irrigação e são 
decisivos no planejamento e na operação desses sistemas (OLIVEIRA \& VILLAS BÔAS, 2008).

Para a avaliação da uniformidade de aplicação da água, podem ser utilizados coeficientes de uniformidade de Chistiansen (CUC), o coeficiente uniformidade estatístico (CUE), o coeficiente de uniformidade de distribuição (CUD), o coeficiente de uniformidade absoluto (CUA), o coeficiente de uniformidade de Hart (CUH) e a eficiência padrão (UDH).

A distinção entre os coeficientes de distribuição ocorre em variação que pode influenciar na observação da ocorrência de distúrbios de vazão, e consequentemente no manejo do sistema de irrigação. Deste modo, objetivou-se neste estudo avaliar a uniformidade de aplicação por meio dos diferentes coeficientes de uniformidade (coeficiente de Christiansen - CUC; estatístico - CUE; distribuição - CUD; absoluto - CUA; Hart CUH; e a eficiência padrão de HSPA) em sistema de irrigação por gotejamento em condição superficial e subsuperficial.

\section{MATERIAL E MÉTODOS}

O experimento foi conduzido durante o ano de 2012, em bancada de ensaio instalada em casa de vegetação na área experimental do Instituto Federal de Educação, Ciência e Tecnologia Goiano - Campus Rio Verde. A casa de vegetação é constituída de cobertura de filme plástico polietileno transparente, de 150 micras e laterais fechadas, com tela tipo sombrite com 30\% de interceptação.

O delineamento experimental utilizado foi inteiramente casualizado analisado em esquema de parcelas subdividas 6 × 20, com 10 repetições, as parcelas eram compostas por seis distribuições de partículas sólidas e as subparcelas 20 ensaios. A linha comportava 60 emissores dos quais foram selecionados aleatoriamente 10 para a realização dos ensaios; sendo deste modo realizados 20 ensaios durante um ano.

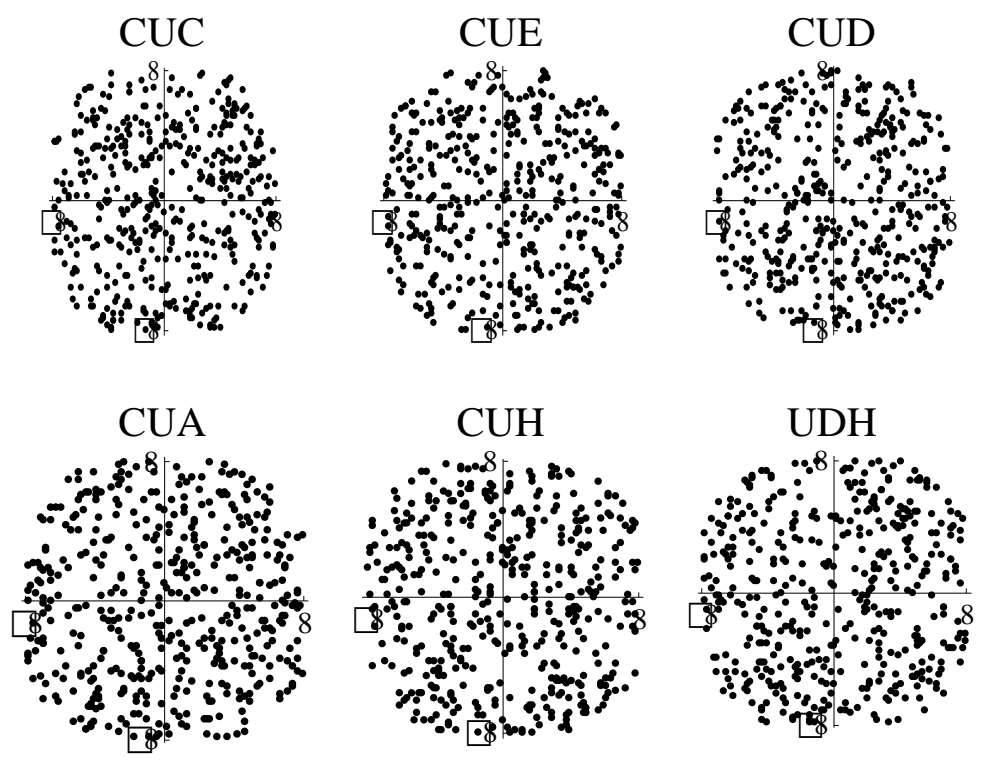

Figura 1. Distribuição de partículas sólidas no interior dos emissores (diâmetro nominal).

Foi utilizado um modelo de tubo gotejador com vazão nominal de $1 \mathrm{~L} \mathrm{~h}^{-1}$, diâmetro nominal 16 mm, diâmetro interno 13 mm, pressão de operação 100 a 350 kpa e espaçamento entre emissores de 0,5 m. O sistema de pressurização utilizado no experimento foi composto por uma motobomba centrífuga.

À entrada das linhas gotejadoras foi instalada uma tomada de pressão, permitindo que a cada medição de vazão a pressão fosse checada e, se necessário, ajustada àquela préestabelecida. Para isso, foi utilizado um manômetro de bourdon com faixa de leitura de $0-4 \mathrm{Kgf}^{\mathrm{cm}}{ }^{-2}$. Durante todo o período do ensaio foram realizadas leituras de temperatura da água no reservatório de captação.

Para a realização dos ensaios, foi retirado o sistema de filtros para que ocorresse a 
redução progressiva a passagem da água nos emissores, devido à presença de sedimentos sólidos e ao desenvolvimento de colônias bacterianas, além disso não foi realizada a limpeza do reservatório favorecendo o aparecimento de algas; assim com essa tendência natural e constante a obstrução, foram realizados os ensaios. Durante todo o período do ensaio foram realizadas leituras de temperatura da água no reservatório de captação; com temperatura da água mantida em $25{ }^{\circ} \mathrm{C}\left(25{ }^{\circ} \mathrm{C} \pm 1{ }^{\circ} \mathrm{C}\right)$.

Para observar a uniformidade de distribuição com os entupimentos, quando necessário, os gotejadores foram lacrados de maneira progressiva, (formando uma progressão aritmética de razão 1,58\%, com entupimentos chegando a no máximo a 30\% do total de emissores avaliados) por meio do uso de adesivo plástico à saída do orifício dos emissores, de forma uniformemente distribuída ao longo da linha lateral conforme metodologia adotada por Bralts et al. (1981). O procedimento de aplicação de água consistiu de 6 horas diária.

A vazão foi medida em um total de 60 emissores (fator distribuição de partículas sólidas), para deste modo ser estabelecida a uniformidade de distribuição de água no sistema de irrigação por gotejamento em condição superficial e subsuperficial, sendo a avaliação do sistema de gotejamento superficial e subsuperficial independentes, quaisquer comparação entre as condições foi baseada na classificação proposta pela ASAE (2008) e Mantovani (2001).

Para avaliação do sistema de gotejamento subsuperficial, a unidade experimental (dispositivo de medição de vazão) foi constituída de recipientes de PVC com as seguintes dimensões: 0,1 m x 0,6 m (D x C), contendo cada recipiente um gotejador. Em condição subsuperficial, os gotejadores foram instalados a 0,20 $\mathrm{m}$ de profundidade dentro do dispositivo.

Para o sistema de gotejamento superficial e subsuperficial os ensaios iniciaram-se após 1 hora de funcionamento do sistema, para que assim fosse garantida a saturação das colunas de solo (gotejamento subsuperficial), sendo em seguida realizada a leitura de vazão.

O procedimento para realização da leitura de vazão consistiu da pressurização do sistema, estabilização da pressão em 150 kPa (+/- 5 kPa) no início da linha, posicionamento dos coletores sob os respectivos gotejadores com três segundos de defasagem e retirada dos coletores com a mesma sequência e defasagem de tempo após cinco minutos de coleta.

Para se obter maior exatidão foi utilizado o método gravimétrico para a determinação do volume coletado de cada emissor, expressandose os valores de vazão em $\mathrm{L} \mathrm{h}^{-1}$, utilizando para tal uma balança de precisão certificada (OHAUS) com precisão de 0,01 g.

O monitoramento da vazão dos gotejadores $\left(\mathrm{L} \mathrm{h}^{-1}\right)$ permitiu a obtenção da vazão média dos gotejadores em condição superficial e subsuperficial em cada ensaio utilizando-se a equação 1.

$$
q=\frac{M}{1000 \times t} 60
$$

em que:

$q$ - vazão do gotejador, $\mathrm{L} \mathrm{h}^{-1}$;

$M$ - massa de água coletada, g; e

$t$ - tempo de coleta, min.

Foram efetuados os cálculos da vazão e de uniformidade de aplicação de água pelos métodos mais utilizados na literatura, destacados nas equações 2 a 7 . 


$$
C U C=100\left\{1-\frac{\sum_{i=1}^{n}\left|X_{i}-\bar{X}\right|}{n \cdot \bar{X}}\right\}
$$

CUC - Coeficiente de uniformidade de Christiansen (CHRISTIANSEN, 1942), em \%.

$$
C U E=100\left(1-\frac{S}{\bar{X}}\right)
$$

CUE - Coeficiente de uniformidade estatístico (WILCOX \& SWAILES, 1947), em \%.

$$
C U D=100\left(\frac{X_{25 \%}}{\bar{X}}\right)
$$

CUD - Coeficiente de uniformidade de distribuição (CRIDDLE et al., 1956), em \%.

$$
C U A=50\left(\frac{X_{25 \%}}{\bar{X}}+\frac{\bar{X}}{X_{12,5 \%}}\right)
$$

CUA - Coeficiente de uniformidade absoluto (KARMELI \& KELLER, 1975), em \%.

$$
C U H=100\left\{1-\sqrt{\frac{2}{\pi}}\left(\frac{S}{\bar{X}}\right)\right\}
$$

CUH - Coeficiente de uniformidade de Hart (HART, 1961), em \%.

$$
U D H=100\left(1-1,27 \frac{S}{\bar{X}}\right)
$$

UDH - Eficiência padrão da HSPA (HART, 1961), em \%.

em que:

$X_{i}$ - vazão de cada gotejador, em $\mathrm{L} \mathrm{h}^{-1}$;

$\bar{X}$ - vazão média dos gotejadores, em $\mathrm{L} \mathrm{h}^{-1}$;

$n$ - número de gotejadores observados;

$S$ - desvio-padrão dos dados de vazão, em $\mathrm{L} \mathrm{h}^{-1}$;
$X_{25 \%}$ - média de $25 \%$ do total de gotejadores, com as menores vazões, em $\mathrm{L} \mathrm{h}^{-1}$; e

$X_{12,5 \%}$ - média de 12,5\% do total de gotejadores, com as menores vazões, em $\mathrm{L} \mathrm{h}^{-1}$.

Para avaliação dos coeficientes de uniformidade utilizou-se as classificações dos valores encontrados propostas na literatura para cada coeficiente - CUC, CUE e CUD (MANTOVANI, 2001); CUA, CUH e UDH (ASAE, 2008).

Os dados obtidos foram submetidos à análise de variância pelo teste $\mathrm{F}$ ao nível de $1 \mathrm{e}$ $5 \%$ de probabilidade, e em caso de significância, os dados foram interpretados mediante regressão polinomial linear e quadrática e as médias foram comparadas entre si pelo teste Tukey à 5\% de probabilidade.

\section{RESULTADOS E DISCUSSÃO}

Na Figura 2 são apresentados os dados de vazão em função dos ensaios realizados para os sistemas de gotejamento em condição superficial e subsuperficial. A discrepância de vazão em relação a vazão nominal dos emissores se mostrou maior em condição subsuperficial, apresentando, desta maneira uma maior amplitude na variação em praticamente todos os ensaios realizados, o que é provavelmente devido a sucção de partículas do solo via gotejador, similarmente Cunha et al. (2013) observaram que os gotejadores tenderam a apresentar redução de vazão quando enterrados, os quais não demonstraram possuir mecanismos que impedissem efetivamente a entrada de partículas sólidas em seu interior. 


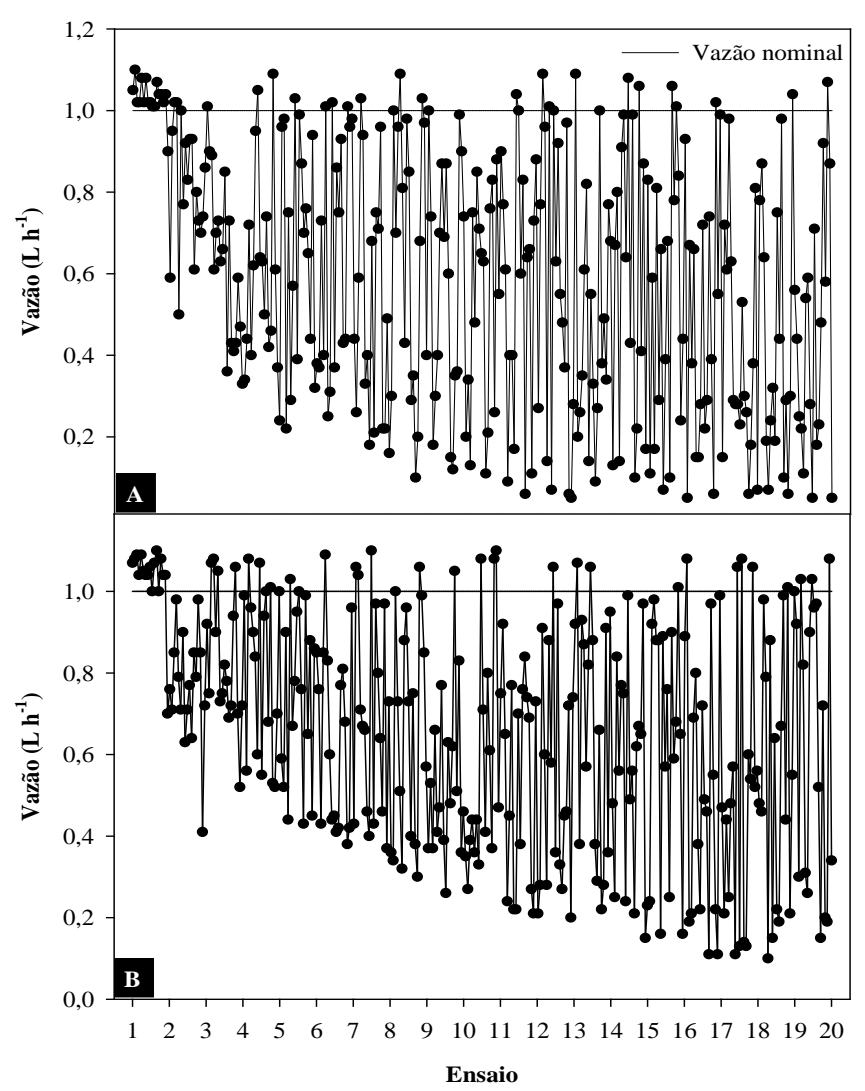

Figura 2. Vazão dos emissores de acordo com o ensaio realizado para o sistema de irrigação por gotejamento. A - Subsuperficial. B - Superficial.

Do ponto de vista prático, a vazão média de emissores pode ser considerada bom parâmetro para avaliar alterações quanto ao funcionamento adequado de emissores, seja devido a problemas de entupimento, seja a outros problemas (CARARO et al., 2006; MÉLO, 2007); logo verifica-se através da vazão obtida que, para o modelo avaliado, a água foi melhor distribuída quando os gotejadores se encontravam superficialmente.

A manutenção no subsolo é mais difícil; a taxa de vazão de cada emissor pode ser afetada pelas propriedades do solo (GIL et al., 2008), além da possibilidade de entupimento por partículas sólidas e raízes (SOUZA et al., 2012).

A deposição do material inicial quando em obstrução parcial tem pouca diferenciação na uniformidade devido à redução de vazão de muitos emissores ainda ser discreta, sendo consequentemente mais preponderante para a determinação da obstrução total (Figura 1), pois a vazão neste caso é afetada de forma bastante significativa.

As médias dos resultados de uniformidade de aplicação de água calculados por diferentes métodos propostos para cada ensaio em sistema de gotejamento superficial são apresentadas na Tabela 1. Observa-se nos primeiros ensaios pouca diferença significativa entre os diferentes métodos. Porém, ao proceder com os ensaios, ocorre uma diferenciação nos resultados da uniformidade, obtendo semelhança apenas entre o coeficiente de uniformidade estatístico (CUE) e o coeficiente de uniformidade absoluto (CUA), apesar de diferirem no último ensaio.

Tabela1. Uniformidade de aplicação de água calculada por diferentes métodos para ensaios realizados em sistema de gotejamento superficial.

\begin{tabular}{|c|c|c|c|c|c|c|c|c|c|c|c|c|}
\hline \multirow{3}{*}{$\begin{array}{c}\text { Ensaio } \\
1\end{array}$} & \multicolumn{12}{|c|}{ Coeficientes de Uniformidade } \\
\hline & \multicolumn{2}{|c|}{ CUC } & \multicolumn{2}{|c|}{ CUE } & \multicolumn{2}{|c|}{ CUD } & \multicolumn{2}{|c|}{ CUA } & \multicolumn{2}{|c|}{ CUH } & \multicolumn{2}{|c|}{ UDH } \\
\hline & 97,67 & $\mathrm{a}$ & 97,10 & a & 96,65 & $\mathrm{a}$ & 96,04 & $\mathrm{a}$ & 86,45 & $\mathrm{~b}$ & 96,32 & a \\
\hline 2 & 89,24 & $\mathrm{a}$ & 87,03 & a & 83,54 & $\mathrm{~b}$ & 83,96 & $\mathrm{~b}$ & 71,27 & c & 83,52 & b \\
\hline 3 & 82,52 & $\mathrm{a}$ & 78,95 & b & 76,07 & c & 75,86 & c & 63,43 & d & 73,27 & c \\
\hline 4 & 78,26 & $\mathrm{a}$ & 74,13 & b & 67,96 & d & 70,69 & c & 59,49 & e & 67,14 & d \\
\hline 5 & 74,77 & $\mathrm{a}$ & 69,94 & $\mathrm{~b}$ & 63,32 & d & 65,97 & c & 56,26 & e & 61,82 & $\mathrm{~d}$ \\
\hline 6 & 71,87 & $\mathrm{a}$ & 66,17 & b & 61,48 & C & 63,59 & C & 53,62 & e & 57,04 & d \\
\hline 7 & 68,43 & $\mathrm{a}$ & 62,23 & b & 53,48 & d & 58,59 & C & 51,07 & e & 52,03 & d \\
\hline 8 & 66,46 & $\mathrm{a}$ & 60,12 & b & 49,07 & C & 57,04 & b & 49,63 & C & 49,36 & c \\
\hline 9 & 63,28 & $\mathrm{a}$ & 57,32 & b & 46,83 & d & 54,65 & C & 47,89 & d & 45,80 & d \\
\hline 10 & 62,44 & $\mathrm{a}$ & 56,37 & b & 47,45 & d & 54,71 & C & 47,31 & d & 44,58 & e \\
\hline 11 & 61,37 & $\mathrm{a}$ & 55,40 & b & 48,35 & C & 54,37 & b & 46,72 & C & 43,36 & d \\
\hline 12 & 61,09 & a & 54,44 & b & 44,00 & d & 52,56 & b & 46,16 & C & 42,15 & d \\
\hline 13 & 58,56 & $\mathrm{a}$ & 52,70 & b & 41,2 & d & 51,78 & b & 45,17 & C & 39,93 & e \\
\hline 14 & 58,02 & $\mathrm{a}$ & 51,03 & b & 37,47 & d & 48,67 & b & 44,17 & C & 37,80 & d \\
\hline 15 & 56,82 & a & 49,27 & b & 39,50 & d & 48,82 & b & 43,22 & C & 35,58 & e \\
\hline 16 & 55,61 & $\mathrm{a}$ & 46,93 & b & 37,82 & d & 46,55 & b & 41,88 & C & 32,60 & e \\
\hline 17 & 54,88 & $\mathrm{a}$ & 46,99 & b & 37,63 & d & 46,51 & b & 41,92 & C & 32,67 & e \\
\hline 18 & 53,24 & $\mathrm{a}$ & 45,94 & b & 37,66 & d & 47,17 & b & 41,34 & C & 31,34 & e \\
\hline 19 & 50,34 & $\mathrm{a}$ & 42,40 & b & 32,54 & d & 43,77 & b & 39,49 & C & 26,85 & e \\
\hline 20 & 46,73 & $\mathrm{a}$ & 37,33 & c & 34,59 & d & 42,47 & b & 36,92 & C & 20,41 & e \\
\hline $\bar{X}$ & 65,58 & & 59,59 & & 51,83 & & 58,19 & & 50,67 & & 48,68 & \\
\hline CV\% & 4,31 & & 5,68 & & 9,77 & & 4,80 & & 4,01 & & $\begin{array}{l}8,82 \\
\end{array}$ & \\
\hline
\end{tabular}

*Média com a mesma letra minúscula na linha entre os métodos não indica diferença significativa pelo teste Tukey, a 5\% de probabilidade. 
O coeficiente que demonstrou a menor uniformidade entre os ensaios foi o coeficiente de eficiência padrão da HSPA (UDH), obtendo uma média de 48,68\%. Já a maior variação foi observada para o coeficiente de uniformidade de distribuição (CUD), com um coeficiente de variação de 9,77\% entre os ensaios. O coeficiente de uniformidade de Christiansen (CUC) se destacou com a maior média dentre os demais coeficientes, com 65,58\%, demonstrando uma menor variação em se tratando do primeiro e último ensaio (Tabela 1).

Os coeficientes quando comparados entre si, dos maiores para os menores percentuais superficialmente, apresentaram a seguinte classificação: CUA, CUC, CUE, CUH, UDH e CUD.

Na Tabela 2, são apresentadas as médias dos resultados de uniformidade de aplicação de água calculados para cada ensaio, por diferentes métodos propostos em sistema de gotejamento subsuperficial. Os coeficientes que proporcionaram menor variação entre o primeiro e o último ensaio foram o coeficiente de uniformidade de Christiansen (CUC) e o coeficiente de uniformidade absoluto (CUA), com média 56,02 e 49,88\% respectivamente. Os resultados dos dois métodos foram semelhantes quanto à distribuição ao longo dos ensaios, apresentando média análoga estatisticamente no último teste.
Tabela 2. Uniformidade de aplicação de água calculada por diferentes métodos para ensaios realizados em sistema de gotejamento subsuperficial.

\begin{tabular}{|c|c|c|c|c|c|c|c|c|c|c|c|c|}
\hline \multirow{3}{*}{$\begin{array}{c}\text { Ensaio } \\
1\end{array}$} & \multicolumn{12}{|c|}{ Coeficientes de Uniformidade } \\
\hline & \multicolumn{2}{|c|}{ CUC } & \multicolumn{2}{|c|}{ CUE } & \multicolumn{2}{|c|}{ CUD } & \multicolumn{2}{|c|}{ CUA } & \multicolumn{2}{|c|}{ CUH } & \multicolumn{2}{|c|}{ UDH } \\
\hline & 97,77 & $\mathrm{a}$ & 97,27 & a & 96,68 & $\mathrm{a}$ & 96,43 & $\mathrm{a}$ & 86,83 & $\mathrm{~b}$ & 96,53 & $\mathrm{a}$ \\
\hline 2 & 83,89 & $\mathrm{a}$ & 80,28 & $\mathrm{~b}$ & 76,32 & c & 77,049 & c & 64,58 & d & 74,96 & c \\
\hline 3 & 77,25 & $\mathrm{a}$ & 71,88 & $\mathrm{a}$ & 65,72 & $\mathrm{~b}$ & 67,67 & $\mathrm{~b}$ & 57,74 & c & 64,29 & $\mathrm{~b}$ \\
\hline 4 & 72,10 & $\mathrm{a}$ & 65,88 & $\mathrm{~b}$ & 58,37 & c & 62,53 & $\mathrm{~b}$ & 53,44 & d & 56,66 & c \\
\hline 5 & 62,54 & $\mathrm{a}$ & 57,09 & $\mathrm{~b}$ & 47,00 & d & 55,59 & c & 47,75 & d & 45,51 & d \\
\hline 6 & 58,72 & $\mathrm{a}$ & 52,79 & $\mathrm{~b}$ & 45,75 & c & 52,49 & $\mathrm{~b}$ & 45,19 & c & 40,04 & d \\
\hline 7 & 55,25 & $\mathrm{a}$ & 48,34 & b & 41,93 & c & 48,95 & $\mathrm{~b}$ & 42,68 & c & 34,39 & d \\
\hline 8 & 53,88 & a & 45,60 & b & 37,35 & d & 46,34 & $\mathrm{~b}$ & 41,18 & c & 30,91 & e \\
\hline 9 & 53,17 & a & 47,50 & b & 35,84 & d & 46,96 & $\mathrm{~b}$ & 42,19 & c & 33,32 & e \\
\hline 10 & 51,09 & a & 40,15 & b & 32,26 & d & 41,48 & $\mathrm{~b}$ & 38,31 & c & 23,99 & e \\
\hline 11 & 50,98 & a & 42,65 & b & 34,51 & d & 43,83 & $\mathrm{~b}$ & 39,58 & c & 27,17 & e \\
\hline 12 & 50,33 & a & 42,13 & b & 27,33 & d & 42,22 & $\mathrm{~b}$ & 39,31 & C & 26,50 & d \\
\hline 13 & 47,74 & $\mathrm{a}$ & 38,03 & c & 29,57 & d & 41,42 & $\mathrm{~b}$ & 37,27 & c & 21,30 & e \\
\hline 14 & 47,34 & a & 39,65 & c & 27,23 & d & 41,18 & $\mathrm{~b}$ & 38,02 & c & 23,35 & e \\
\hline 15 & 46,94 & $\mathrm{a}$ & 39,54 & c & 29,73 & d & 41,91 & $\mathrm{~b}$ & 37,99 & c & 23,22 & e \\
\hline 16 & 45,66 & $\mathrm{a}$ & 33,92 & c & 29,71 & d & 38,69 & $\mathrm{~b}$ & 35,20 & c & 16,07 & e \\
\hline 17 & 43,99 & $\mathrm{a}$ & 33,83 & c & 28,93 & d & 39,02 & $\mathrm{~b}$ & 35,13 & c & 15,97 & e \\
\hline 18 & 42,18 & $\mathrm{a}$ & 35,83 & $\mathrm{~b}$ & 29,48 & c & 41,59 & $\mathrm{a}$ & 36,09 & $\mathrm{~b}$ & 18,50 & d \\
\hline 19 & 40,94 & $\mathrm{a}$ & 31,13 & d & 24,33 & e & 36,26 & $\mathrm{~b}$ & 33,79 & c & 12,54 & $\mathrm{f}$ \\
\hline 20 & 38,57 & $\mathrm{a}$ & 30,92 & c & 21,91 & d & 35,99 & $\mathrm{a}$ & 33,69 & b & 12,27 & e \\
\hline $\bar{X}$ & 56,02 & & 48,72 & & 41,00 & & 49,88 & & 44,30 & & 34,87 & \\
\hline CV\% & 4,28 & & 7,66 & & 11,04 & & 6,10 & & 4,56 & & 13,58 & \\
\hline
\end{tabular}

*Média com a mesma letra minúscula na linha entre os métodos não indica diferença significativa pelo teste Tukey, a 5\% de probabilidade.

Os coeficientes que apresentaram as maiores variações foram o coeficiente de eficiência padrão da HSPA (UDH) e o coeficiente de uniformidade de distribuição (CUD), com coeficientes de variação de 13,58 e $11,04 \%$ respectivamente. 
O CUC em condição subsuperficial foi mais sensível aos distúrbios de vazão, diferentemente do que ocorreu no gotejamento superficial, já que nesta condição teve como destaque o CUA, novamente o CUD foi menos sensível. Keller e Karmeli (1974) considera o CUD o mais rigoroso para o dimensionamento e a avaliação em campo de sistema de irrigação, entretanto mesmo sendo o CUD mais rigoroso, este não apresenta uma boa discriminação e logo não identifica de maneira adequada os distúrbios de vazão. Os coeficientes quando comparado entre si, dos maiores para os menores percentuais subsuperficialmente, devido à maior ocorrência de distúrbios de vazão, apresentaram a seguinte classificação: CUC, CUA, CUH, CUE, UDH e CUD; comparando esta classificação com a ordem obtida quando em condição superficial pode-se verificar duas trocas de posição entre CUA e CUC, e entre CUE e CUH.

De maneira geral o CUC (subsuperficial) e o CUA (superficial) foram os que apresentaram maior sensibilidade no que diz respeito à uniformidade, conseguindo identificar 62,5\% dos distúrbios de vazões, enquanto que o CUE, CUH e UDH distinguiram 56,25 e 50\%, superficialmente e subsuperficialmente respectivamente, já o CUA teve uma queda mais expressiva de $12,5 \%$ de uma condição para outra; de maneira diferente foi observado para o CUD que mostrou um aumento da diferenciação dos ensaios, da condição superficial para a subsuperficial de $37,5 \%$, para $43,75 \%$ mesmo assim este percentual ficou abaixo do esperado ou do mínimo tido como adequado de 50\%; o mesmo foi observado para o CUC que de uma condição para a outra apresentou um acréscimo de 6,25\%, logo se verifica que o coeficiente de uniformidade de Christiansen demonstra um melhor desempenho discriminativo médio, nos dois sistemas de irrigação avaliados (superficial e subsuperficial).

A Figura 3 apresenta a distribuição dos resultados de uniformidade de aplicação de água dos coeficientes em função dos ensaios realizados. Observa-se uma queda acentuada da uniformidade nos ensaios iniciais, assim bem como uma redução de uniformidade nos testes finais.

Todos os resultados dos coeficientes de uniformidade se adequaram em coeficientes quadráticos (significante ao nível de $1 \%$ de probabilidade), com $\mathrm{R}^{2}$ variando entre $86 \mathrm{e}$ 97\%. Destaca-se que o coeficiente de uniformidade de Christiansen (CUC) proporcionou uma sequência de resultados com menor variação ao longo dos testes, para ambos os sistemas de gotejamento (superficial e subsuperficial) (Figura 3A).

Os resultados de uniformidade de aplicação de água do método de eficiência padrão da HSPA (UDH) e do coeficiente de uniformidade de distribuição (CUD) demonstram maior variação ao longo dos ensaios, principalmente para o sistema de gotejamento subsuperficial, demonstrando menor eficiência para este sistema (Figuras 3C e 3F), com base na classificação proposta por Mantovani (2001) e ASAE (2008). 

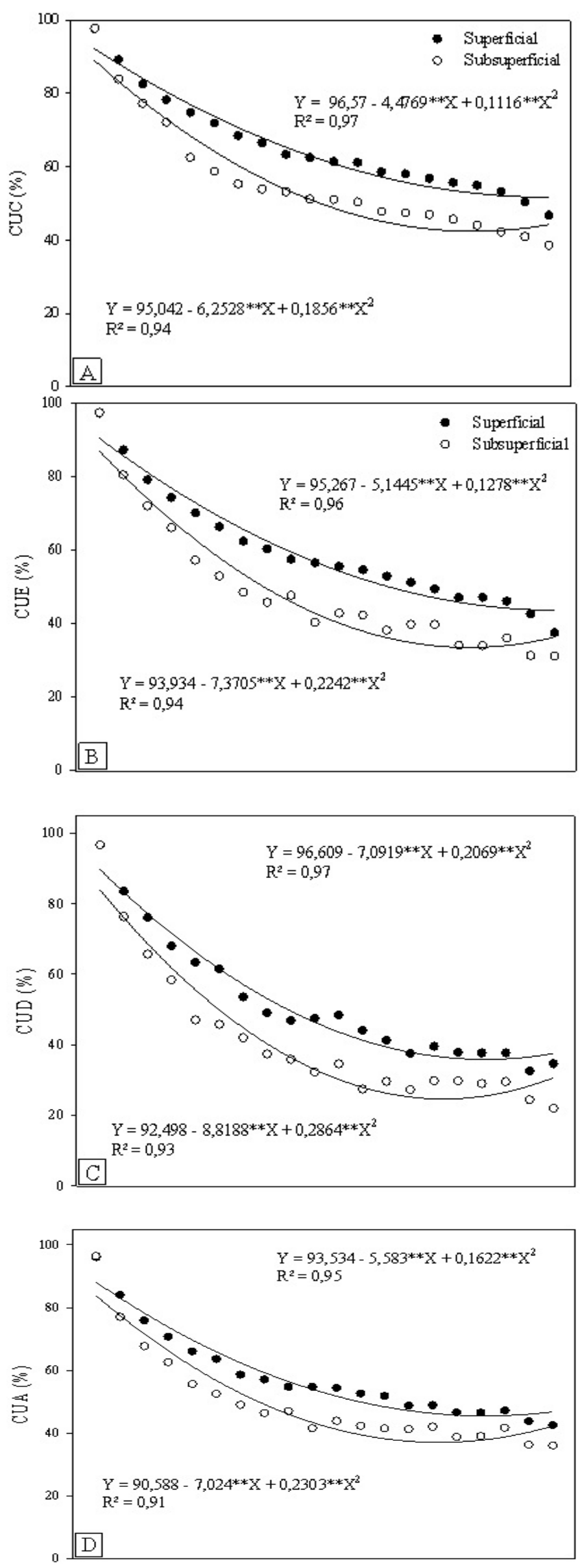
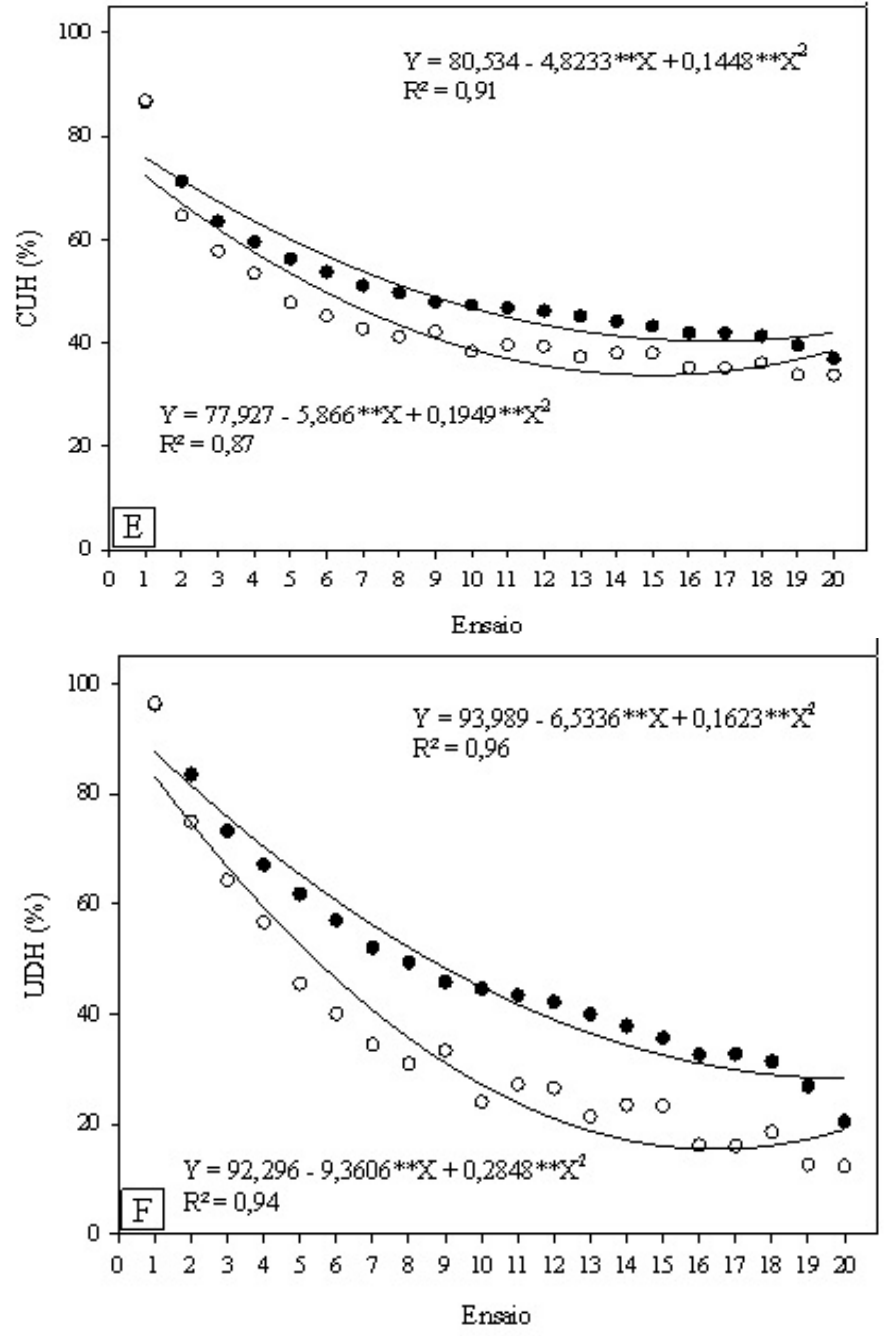

Figura 3. Coeficientes de uniformidade de Christiansen (A), estatístico (B), distribuição (C), absoluto (D), Hart (E) e eficiência padrão da HSPA (F) para cada ensaio no sistema de gotejamento em condição superficial e subsuperficial.

O CUD e o UDH dentre os coeficientes avaliados apresentaram os menores valores, entretanto em sistemas de irrigação localizada, segundo López et al (1992) e Frizzone \& Dourado Neto (2003), o CUD é mais utilizado na avaliação, pois este possibilita uma medida mais restrita, dando maior peso às plantas que recebem menos água, sendo o mesmo adotado para o cálculo do tempo de irrigação; nesse sentido, o UDH poderia ser mais interessante, por ser mais restrito e também por apresentar uma melhor discriminação, em ampla variação de vazões (condições adversas) e segundo 
Cunha et al. (2009) quando a lâmina de irrigação aplicada tem distribuição normal, o CUD é igual à UDH.

\section{CONCLUSÃO}

O CUA foi mais sensível em condição superficial enquanto o CUC teve maior discriminação em condição subsuperficial e foram os que apresentaram maior sensibilidade no que diz respeito à uniformidade, conseguindo identificar $62,5 \%$ dos distúrbios de vazões.

O CUD não identifica de maneira adequada os distúrbios de vazão, com uma baixa discriminação na uniformidade.

Os coeficientes quando comparado entre si, dos maiores para os menores percentuais de discriminação superficialmente, apresentaram a seguinte classificação: CUA, CUC, CUE, CUH, UDH e CUD; já subsuperficialmente CUC, CUA, CUH, CUE, UDH e CUD.

\section{REFERÊNCIAS BIBLIOGRÁFICAS}

ASAE. ASAE EP 405.1: standards, design and installation of microirrigation systems. [S.l.], 2008.

BARRETO FILHO, A. A.; DANTAS NETO, J.; MATOS, J. A.; GOMES, E. M.; Desempenho de um sistema de irrigação por microaspersão, instalado a nível de campo. Revista Brasileira Engenharia Agrícola e Ambiental, Campina Grande, v. 4, n. 3, p. 309314, 2000.

BARROS, A. C.; FOLEGATTI, M. V.; SOUZA, C. F.; SANTORO, L. B. Distribuição de água no solo aplicado por gotejamento enterrado e superficial. Revista Brasileira de
Engenharia Agrícola e Ambiental, Campina Grande, v. 13, n. 6, p. 700-707, 2009.

BERNARDO, S.; SOARES, A. A.; MANTOVANI, E. C. Manual de irrigação. 8ed. Viçosa: UFV, 2008. 625 p.

BRALTS, V. F.; WU, I. P.; GITLIN, H. M. Drip irrigation uniformity considering emitter plugging. Trans ASAE 24:1234, 1981.

CARARO, D. C.; BOTREL, T. A.; HILLS, D. J.; LEVERENZ, H. L. Analysis of clogging in drip emitters during wastewater irrigation. Applied Engineering in Agriculture, St. Joseph, v.22, n.2, p.251-257, 2006.

CHRISTIANSEN, J.E. Irrigation by Sprinkling.Berkeley: California Agricultural Station. 1942. 124p. Bulletin, 670.

CRIDDLE, W.D.; DAVIS, S.; PAIR, C.H.; SHOCKLEY, D.G. Methods for Evaluating Irrigation Systems. Washington DC: Soil Conservation Service - USDA, Agricultural Handbook, 82, 1956. 24p..

CUNHA, F. F.; ALENCAR, C. A. B.; VICENTE, M. R.; BATISTA R. O.; SOUZA, J. A. R. Comparação de equações para cálculo da uniformidade de aplicação de água para diferentes sistemas de irrigação. Engenharia na agricultura, Viçosa, v.17, n.5, 2009.

CUNHA, F. N.; SILVA, N. F.; OLIVEIRA, R. C.; TEIXEIRA, M. B.; CARVALHO, J. J.; GOMES, R. R. F. Caracterização hidráulica de gotejadores em condição superficial e subsuperficial. Fortaleza, CE, INOVAGRI. Revista Brasileira de Agricultura Irrigada v.7, no. 5, p 317 - 329, 2013.

FARIA, L. F.; COELHO, R. D.; RESENDE, R. S. Variação de vazão de gotejadores de fluxo normal enterrados na irrigação de café. 
Engenharia Agrícola, Jaboticabal, v. 24, n. 3, p. 589-602, 2004.

FRIZZONE, J. A.; DOURADO NETO, D. Avaliação de sistemas de irrigação. In: MIRANDA, J. H.; PIRES, R. C. de M. (Org.). Irrigação. Piracicaba, SP: FUNEP, 2003. p. 573-651.

GIL, M.; SINOBAS, R.; JUANA, L.; SÁCHEZ, R.; LOSADA, A. Emitter discharge variability of subsurface drip irrigation in uniform soils: Effect on water-application uniformity. Irrigation Science, v.26, n. 6, p.451-458, 2008.

HART, W.E. Overhead irrigation pattern parameters. Transactions of the ASAE, Saint Joseph, v.42, n.7, p.354-355, 1961.

KARMELI, D.; KELLER, J. Trickle Irrigation Design. Glendora: Rain Bird Manufacturing Corporation, 1975. 132p.

KELLER, J.; KARMELI, D. Trickle irrigation design parameters. Transactions of the ASAE. St. Joseph, v.17, p.678-684,1974.

LÓPEZ, J. R., ABREU, J. M. H.; REGALADO, A.P.; HERNÁNDEZ, J.F.G. Riego Localizado. Madrid, Espana: Mundi Prensa, 1992. 405p.

MANTOVANI, E. C.; FACCIOLI, G. G.; LEAL, B. G.; SOARES, A. A.; COSTA, L. C.;
FREITAS, P. S. L. Influence of the water distribution uniformity and irrigation depth on the yield of irrigated bean crop. Irriga, Botucatu, v. 14, n. 4, p. 458-469, 2009.

MANTOVAni, E. C. AVAliA: Programa de Avaliação da Irrigação por Aspersão e Localizada. Viçosa, MG: UFV. 2001.

MÉLO, R. F. Dinâmica e controle do entupimento de gotejadores em função de precipitados químicos e plâncton. Tese (Doutorado em Irrigação e Drenagem) - Escola Superior de Agricultura "Luiz de Queiroz", Universidade de São Paulo, Piracicaba, 2007, 189p.

SILVA, E. M.; LIMA, J. E. F. W.; AZEVEDO, J. A.; RODRIGUES, L. N. Proposição de um modelo matemático para a avaliação do desempenho de sistemas de irrigação. Pesquisa Agropecuária Brasileira, Brasília, v.39, n.8, p.741-748, 2004.

SOUZA, W. J.; BROTEL, T. A.; COELHO, R. D.; NOVA, N. A. V. Irrigação localizada subsuperficial: gotejador convencional e novo protótipo. Revista Brasileira de Engenharia Agrícola e Ambiental, v. 16, n. 8, p. 811-819, 2012.

WILCOX, J. C.; SWAILES, G. E. Uniformity of water distribution by some under tree orchard sprinklers. Scientific Agriculture, Ottawa, v.27, n.11, p.565-583, 1947. 